\title{
BOUNDS FOR THE DISTRIBUTION OF SOME FUNCTIONALS OF PROCESSES WITH $\varphi$-SUB-GAUSSIAN INCREMENTS
}

UDC 519.21

\author{
R. E. YAMNENKO
}

ABstract. Bounds for the distribution of some functionals of a stochastic process $\{X(t), t \in T\}$ belonging to the class $V(\varphi, \psi)$ are obtained. An example of the functionals studied in the paper is given by

$$
\mathrm{F}\left\{\sup _{s \leq t ; s, t \in B}(X(t)-X(s)-(f(t)-f(s)))>x\right\},
$$

where $f(t)$ is a continuous function that can be viewed as a service output rate of a queue formed by the process $X(t)$. For the latter interpretation, the bounds can be viewed as upper estimates for the buffer overflow probabilities with buffer size $x>0$. The results obtained in the paper apply to Gaussian stochastic processes. As an example, we show an application for the generalized fractional Brownian motion defined on a finite interval.

\section{INTRODUCTION}

This paper continues the line of investigation of queues formed by stochastic processes belonging to the class $V(\varphi, \psi)$ (see, for example, [7, 8, 9]). The classes $V(\varphi, \psi)$ are rather general families of stochastic processes containing Gaussian stochastic processes, among others. Therefore, by studying the processes belonging to the class $V(\varphi, \psi)$, one obtains results for well-known stochastic processes as well as for some of their modern generalizations.

Some extremal functionals of the increments of a stochastic process $\{X(t), t \in T\}$ belonging to the class $V(\varphi, \psi)$ are studied in the paper. In particular, we study the following functionals:

$$
\begin{aligned}
& \sup _{s \leq t ; s, t \in B}(X(t)-X(s)-(f(t)-f(s))), \\
& \inf _{s \leq t ; s, t \in B}(X(t)-X(s)-(f(t)-f(s))), \\
& \sup _{s \leq t ; s, t \in B}|X(t)-X(s)-(f(t)-f(s))|,
\end{aligned}
$$

where $f(t)$ is a certain continuous function. The problems concerning the distribution of such functionals appear, for example, in queue theory. Examples of such problems in queue theory are estimating the maximal length of a queue or the buffer overflow probabilities. The function $f$ above is viewed in queue theory as the service intensity of a queue (see, for example, [1, [5]).

2000 Mathematics Subject Classification. Primary 60G07; Secondary 60K25.

Key words and phrases. Generalized fractional Brownian motion, metric entropy, queue, bounds for the distribution, sub-Gaussian process. 
The paper is organized as follows. Section 1 contains necessary definitions and results of the theory of $\varphi$-sub-Gaussian random variables and stochastic processes. The main results of the paper proved with the help of the method of metric entropy are placed in Section 2. In Section 3, an example of an application of upper bounds obtained in Section 2 is given in the case of sub-Gaussian stochastic processes. In particular, we show that these bounds hold for the fractional Brownian motion.

\section{Stochastic processes belonging to the $\operatorname{spaces} \operatorname{Sub}_{\varphi}(\Omega)$ AND TO THE CLASSES $V(\varphi, \psi)$}

Let $\{\Omega, \mathcal{B}, \mathrm{P}\}$ be a standard probability space and let $T$ be a general set of parameters.

\subsection{Orlicz $N$-functions.}

Definition 1.1 ([2]). A function $U=\{U(x), x \in \mathbb{R}\}$ is called an Orlicz $N$-function if $U$ is continuous, convex, even, and such that $U(0)=0, U(x)$ increases in the set $x>0$, $U(x) / x \rightarrow 0$ as $x \rightarrow 0$, and $U(x) / x \rightarrow \infty$ as $x \rightarrow \infty$.

Condition Q. We say that Condition Q holds for an $N$-function $\varphi$ if

$$
\liminf _{x \rightarrow 0} \frac{\varphi(x)}{x^{2}}=c>0 .
$$

Definition 1.2. We say that an $N$-function $\varphi_{1}$ is subordinated to an $N$-function $\varphi_{2}$ and write $\varphi_{1} \prec \varphi_{2}$ if there are constants $c>0$ and $x_{0}>0$ such that $\varphi_{1}(x)<\varphi_{2}(c x)$ for $x>x_{0}$. We say that two $N$-functions $\varphi_{1}$ and $\varphi_{2}$ are equivalent if both relations $\varphi_{1} \prec \varphi_{2}$ and $\varphi_{2} \prec \varphi_{1}$ hold.

\section{2. $\varphi$-sub-Gaussian random variables and stochastic processes.}

Definition $1.3([2])$. Let $\varphi$ be an $N$-function and let Condition Q hold for $\varphi$. We say that a random variable $\xi$ belongs to the $\operatorname{space} \operatorname{Sub}_{\varphi}(\Omega)$ if $E \xi=0, E \exp \{\lambda \xi\}$ is finite for all $\lambda \in \mathbb{R}$, and there exists a constant $a>0$ such that

$$
\mathrm{E} \exp \{\lambda \xi\} \leq \exp \{\varphi(\lambda a)\}
$$

for all $\lambda \in \mathbb{R}$.

Theorem $1.1([2])$. The space $\operatorname{Sub}_{\varphi}(\Omega)$ is a Banach space with the norm

$$
\tau_{\varphi}(\xi)=\sup _{\lambda>0} \frac{\varphi^{(-1)}(\log \mathrm{E} \exp \{\lambda \xi\})}{\lambda},
$$

where $\varphi^{(-1)}$ denotes the inverse function for $\varphi$. Moreover,

$$
\mathrm{E} \exp \{\lambda \xi\} \leq \exp \left\{\varphi\left(\lambda \tau_{\varphi}(\xi)\right)\right\}
$$

for all $\lambda \in \mathbb{R}$.

Lemma $1.1([8])$. Let $\xi \in \operatorname{Sub}_{\varphi}(\Omega), \tau_{\varphi}(\xi)>0$, and $\varepsilon>0$. Then

$$
\begin{gathered}
\mathrm{P}\{\xi>\varepsilon\} \leq \exp \left\{-\varphi^{*}\left(\frac{\varepsilon}{\tau_{\varphi}(\xi)}\right)\right\}, \\
\mathrm{P}\{\xi<-\varepsilon\} \leq \exp \left\{-\varphi^{*}\left(\frac{\varepsilon}{\tau_{\varphi}(\xi)}\right)\right\}, \\
\mathrm{P}\{|\xi|>\varepsilon\} \leq 2 \exp \left\{-\varphi^{*}\left(\frac{\varepsilon}{\tau_{\varphi}(\xi)}\right)\right\},
\end{gathered}
$$

where

$$
\varphi^{*}(x)=\sup _{y \in \mathbb{R}}(x y-\varphi(y))
$$

is the Young-Fenchel transform of the function $\varphi$. 
Lemma $1.2([])$. Let $\xi \in \operatorname{Sub}_{\varphi}(\Omega)$. Then

$$
\left|\mathrm{E} \xi^{k}\right| \leq \mathrm{E}|\xi|^{k} \leq 2\left(\tau_{\varphi}(\xi)\right)^{k} \frac{e^{k}}{\left(\varphi^{(-1)}(k)\right)^{k}} k !
$$

for $k=1,2, \ldots$.

Example $1.1([2])$. A centered Gaussian random variable $\xi$ belongs to the space

$$
\operatorname{Sub}_{x^{2} / 2}(\Omega) \text {. }
$$

Moreover, $\tau(\xi)=\left(\mathrm{E} \xi^{2}\right)^{1 / 2}$.

Definition 1.4. We say that a stochastic process $X=\{X(t), t \in T\}$ is $\varphi$-sub-Gaussian and write $X(t) \in \operatorname{Sub}_{\varphi}(\Omega)$ if the random variables $X(t), t \in T$, are $\varphi$-sub-Gaussian.

If $\varphi(x)=x^{2} / 2$, then the corresponding stochastic processes bear the name subGaussian.

Example 1.2. A centered Gaussian stochastic process is a sub-Gaussian process.

\subsection{Stochastic processes of the class $V(\varphi, \psi)$.}

Definition $1.5(8])$. Let $\varphi \prec \psi$ be two Orlicz $N$-functions. We say that a stochastic process $X=\{X(t), t \in T\}$ belongs to the class $V(\varphi, \psi)$ if, for all $t \in T$, the random variables $X(t)$ belong to the space $\operatorname{Sub}_{\psi}(\Omega)$ and the increments $(X(t)-X(s))$ belong to

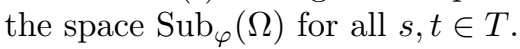

Example $1.3([2])$. If $\varphi(x)=x^{2} / 2$, then all sub-Gaussian processes belong to the class $V(\varphi, \varphi)$.

Example $1.4([8])$. Let

$$
X(t)=\xi_{0}+\sum_{k=1}^{\infty} \xi_{k} f_{k}(t),
$$

where the random variables $\xi_{0} \in \operatorname{Sub}_{\psi}(\Omega)$ and $\left\{\xi_{k}, k=1,2, \ldots\right\} \in \operatorname{Sub}_{\varphi}(\Omega)$ are such that

$$
\sum_{k=1}^{\infty} \tau_{\varphi}\left(\xi_{k}\right)\left|f_{k}(t)\right|<\infty
$$

Then the stochastic process $X(t)$ belongs to the class $V(\varphi, \psi)$.

\section{MAin RESUltS}

Let $(T, \rho)$ be a separable pseudometric (metric) space equipped with a pseudometrie1 (metric) $\rho$.

Consider a separable stochastic process $X=\{X(t), t \in T\}$ with $\varphi$-sub-Gaussian increments. In particular, $X$ may belong to the class $V(\varphi, \psi)$, where $\varphi \prec \psi$. Assume that there exists a continuous increasing function $\sigma=\{\sigma(h), h>0\}$ such that $\sigma(h) \rightarrow 0$ as $h \rightarrow 0$ and

$$
\sup _{\rho(t, s) \leq h} \tau_{\varphi}(X(t)-X(s)) \leq \sigma(h) .
$$

Note that the function

$$
\sigma(h)=\sup _{\rho(t, s) \leq h} \tau_{\varphi}(X(t)-X(s))
$$

possesses this property if the process $X(t)$ is continuous in the norm $\tau_{\varphi}(\cdot)$.

\footnotetext{
${ }^{1} \mathrm{~A}$ pseudometric possesses all the properties of a metric except for the following one: if $\rho(t, s)=0$, then $t=s$. This means that the set $\{(t, s): \rho(t, s)=0\}$ can be wider than the diagonal $\{(t, s): t=s\}$ in the case of a pseudometric (see [2]).
} 
Let $B$ be a compact set, $B \subseteq T$. In what follows we use the following notation: $\beta>0$ is a number such that

$$
\beta \leq \sigma\left(\inf _{s \in B} \sup _{t \in B} \rho(t, s)\right) ;
$$

$\zeta_{\varphi}(v)=v / \varphi^{(-1)}(v) ; N(u)=N_{(B, \rho)}(u)$ is the metric capacity of the space $(B, \rho)$ (in other words, $N(u)$ is the minimal number of closed balls of radius $u$ that cover the space $(B, \rho))$;

$$
L(u)=\frac{1}{2}\left((N(u))^{2}+N(u)\right) \text {. }
$$

Assume that a stochastic process $X$ is defined in a compact set $B \subseteq T$. The following assertions contain sufficient conditions for the boundedness of the supremum

$$
\sup _{s \leq t ; s, t \in B}(X(t)-X(s)-(f(t)-f(s)))
$$

and some bounds for its exponential moment where $f=\{f(t), t \in B\}$ is some continuous function.

Lemma 2.1. Assume that condition (6) holds for a stochastic process

$$
X(t)=\{X(t), t \in B\}
$$

belonging to the class $V(\varphi, \psi)$. Let $f=\{f(t), t \in B\}$ be a continuous function such that

$$
|f(u)-f(v)| \leq \delta(\rho(u, v)),
$$

where $\delta=\{\delta(u), u>0\}$ is a certain increasing nonnegative function. Let a sequence $\left\{q_{k}\right\}_{k=1}^{\infty}$ be such that

$$
q_{k}>1 \quad \text { and } \quad \sum_{k=1}^{\infty} q_{k}^{-1} \leq 1
$$

Finally, let $\left\{\varepsilon_{k}\right\}_{k=1}^{\infty}$ be a decreasing sequence such that $\varepsilon_{k}>0$ and $\varepsilon_{k} \rightarrow 0$ as $k \rightarrow \infty$. Then

$$
\begin{aligned}
& E \exp \left\{\lambda \sup _{s \leq t ; s, t \in B}(X(t)-X(s)-(f(t)-f(s)))\right\} \\
& \leq W(\lambda) \exp \left\{\sum_{k=2}^{\infty}\left(\frac{1}{q_{k}} \varphi\left(2 q_{k} \lambda \sigma\left(\varepsilon_{k-1}\right)\right)+2 \lambda \delta\left(\varepsilon_{k-1}\right)\right)\right\} \prod_{k=2}^{\infty}\left(L\left(\varepsilon_{k}\right)\right)^{1 / q_{k}}
\end{aligned}
$$

for all $\lambda>0$, where

$$
W(\lambda)=\left(\sum_{l=0}^{N\left(\varepsilon_{1}\right)-1}\left(N\left(\varepsilon_{1}\right)-l\right) \exp \left\{\varphi\left(q_{1} \lambda \sigma\left(2 \varepsilon_{1} l\right)\right)+q_{1} \lambda \delta\left(2 \varepsilon_{1} l\right)\right\}\right)^{1 / q_{1}}
$$

Proof. Denote by $V_{\varepsilon_{k}}$ the set of centers of closed balls of radius $\varepsilon_{k}$ that form the minimal covering of the space $(B, \rho)$. The total number of points in the set $V_{\varepsilon_{k}}$ is equal to $N\left(\varepsilon_{k}\right)$.

Clearly $X(t)$ as well as $X_{f}(t)=X(t)-f(t)$ are separable stochastic processes. Lemma 1.1 and assumption (6) imply that

$$
\begin{aligned}
& \mathrm{P}\{|X(t)-X(s)|>\varepsilon\} \\
& \quad \leq 2 \exp \left\{-\varphi^{*}\left(\frac{\varepsilon}{\tau_{\varphi}(X(t)-X(s))}\right)\right\} \leq 2 \exp \left\{-\varphi^{*}\left(\frac{\varepsilon}{\sigma(\rho(t, s))}\right)\right\}
\end{aligned}
$$

for all $\varepsilon>0$. Thus the processes $X$ and $X_{f}$ are continuous in probability. If a separable stochastic process is defined in $(B, \rho)$ and is continuous in probability, then any countable and everywhere dense set with respect to $\rho$ is a set of separability of this process. 
Therefore $V=\bigcup_{k=1}^{\infty} V_{\varepsilon_{k}}$ is a set of $\rho$-separability of the process $X_{f}$. Moreover,

$$
\sup _{s \leq t ; s, t \in B}\left(X_{f}(t)-X_{f}(s)\right)=\sup _{s \leq t ; s, t \in V}\left(X_{f}(t)-X_{f}(s)\right)
$$

with probability one.

Consider a mapping $\alpha_{n}=\left\{\alpha_{n}(u), n=0,1, \ldots\right\}$ of the set $V$ to $V_{\varepsilon_{n}}$, where $\alpha_{n}(u)$ is a point of the set $V_{\varepsilon_{n}}$ such that $\rho\left(u, \alpha_{n}(u)\right) \leq \varepsilon_{n}$. If $u \in V_{\varepsilon_{n}}$, then $\alpha_{n}(u)=u$. If there are several points in the set $V_{\varepsilon_{n}}$ with the property $\rho\left(u, \alpha_{n}(u)\right) \leq \varepsilon_{n}$, then we choose any of them to preserve the condition $\alpha_{n}(v) \leq \alpha_{n}(u)$ for all $v \leq u, v, u \in V$, and denote this point by $\alpha_{n}(u)$.

The Chebyshev inequality, Lemma 1.1, and assumption (6) imply that

$$
\begin{aligned}
\mathrm{P}\left\{\left|X(u)-X\left(\alpha_{n}(u)\right)\right|>p^{n / 2}\right\} & \leq \frac{\mathrm{E}\left(X(u)-X\left(\alpha_{n}(u)\right)\right)^{2}}{p^{n}} \leq \frac{c^{2} \tau_{\varphi}^{2}\left(X(u)-X\left(\alpha_{n}(u)\right)\right)}{p^{n}} \\
& \leq \frac{c^{2} \sigma^{2}\left(\varepsilon_{n}\right)}{p^{n}}=c^{2} \beta^{2} p^{n}
\end{aligned}
$$

where

$$
c=\frac{2 e}{\varphi^{(-1)}(2)}
$$

The latter inequality means that

$$
\sum_{n=1}^{\infty} \mathrm{P}\left\{\left|X(u)-X\left(\alpha_{n}(u)\right)\right|>p^{n / 2}\right\}<\infty .
$$

The Borel-Cantelli lemma yields $X(u)-X\left(\alpha_{n}(u)\right) \rightarrow 0$ with probability one as $n \rightarrow \infty$. Since the function $f$ is continuous,

$$
X_{f}(u)-X_{f}\left(\alpha_{n}(u)\right) \rightarrow 0 \quad \text { as } n \rightarrow \infty
$$

with probability one. The set $V$ is countable, whence $X(u)-X\left(\alpha_{n}(u)\right) \rightarrow 0$ with probability one as $n \rightarrow \infty$ uniformly in $s$.

Let $s$ and $t$ be two arbitrary points of the set $V, s \leq t$. For all $m \geq 1$, let $s_{m}=\alpha_{m}(s)$, $s_{m-1}=\alpha_{m-1}\left(s_{m}\right), \ldots, s_{1}=\alpha_{1}\left(s_{2}\right)$ and $t_{m}=\alpha_{m}(t), t_{m-1}=\alpha_{m-1}\left(t_{m}\right), \ldots, t_{1}=\alpha_{1}\left(t_{2}\right)$. Then, for all $m \geq 2$,

$$
\begin{gathered}
X_{f}(s)=X_{f}\left(s_{1}\right)+\sum_{k=2}^{m}\left(X_{f}\left(s_{k}\right)-X_{f}\left(s_{k-1}\right)\right)+X_{f}(s)-X_{f}\left(\alpha_{m}(s)\right), \\
X_{f}(t)=X_{f}\left(t_{1}\right)+\sum_{k=2}^{m}\left(X_{f}\left(t_{k}\right)-X_{f}\left(t_{k-1}\right)\right)+X_{f}(t)-X_{f}\left(\alpha_{m}(t)\right)
\end{gathered}
$$

and

$$
\begin{aligned}
X_{f}(t) & -X_{f}(s) \\
\leq & \max _{v \leq u ; u, v \in V_{\varepsilon_{1}}}\left(X_{f}(u)-X_{f}(v)\right) \\
& +\sum_{k=2}^{m} \max _{v \leq u ; u, v \in V_{\varepsilon_{k}}}\left(X_{f}(u)-X_{f}\left(\alpha_{k-1}(u)\right)-X_{f}(v)+X_{f}\left(\alpha_{k-1}(v)\right)\right) \\
& +X_{f}(t)-X_{f}\left(\alpha_{m}(t)\right)-X_{f}(s)+X_{f}\left(\alpha_{m}(s)\right) .
\end{aligned}
$$


Now we derive from (77) and (12) that

$$
\begin{aligned}
& \sup _{s \leq t ; s, t \in V}\left(X_{f}(t)-X_{f}(s)\right) \\
& \leq \liminf _{m \rightarrow \infty}\left(\max _{v \leq u ; u, v \in V_{\varepsilon_{1}}}\left(X_{f}(u)-X_{f}(v)\right)\right. \\
& +\sum_{k=2}^{m} \max _{v \leq u ; u, v \in V_{\varepsilon_{k}}}\left(X_{f}(u)-X_{f}\left(\alpha_{k-1}(u)\right)-X_{f}(v)+X_{f}\left(\alpha_{k-1}(v)\right)\right) \\
& \left.+X_{f}(t)-X_{f}\left(\alpha_{m}(t)\right)-X_{f}(s)+X_{f}\left(\alpha_{m}(s)\right)\right)
\end{aligned}
$$

with probability one.

Now we use the Hölder inequality, (11), (13), and the Fatou lemma to prove that

$$
\begin{aligned}
\mathrm{E} \exp & \left\{\lambda \sup _{s \leq t ; s, t \in V}\left(X_{f}(t)-X_{f}(s)\right)\right\} \\
\leq & \mathrm{E} \liminf _{m \rightarrow \infty} \exp \left\{\lambda\left(\max _{v \leq u ; u, v \in V_{\varepsilon_{1}}}\left(X_{f}(u)-X_{f}(v)\right)+\sum_{k=2}^{m} \max _{v \leq u ; u, v \in V_{\varepsilon_{k}}}(K(u, v))\right)\right\} \\
\leq & \liminf _{m \rightarrow \infty} \mathrm{E} \exp \left\{\lambda\left(\max _{v \leq u ; u, v \in V_{\varepsilon_{1}}}\left(X_{f}(u)-X_{f}(v)\right)+\sum_{k=2}^{m \leq u ; u, v \in V_{\varepsilon_{k}}} \max _{v \leq 1}(K(u, v))\right)\right\} \\
\leq & \liminf _{m \rightarrow \infty}\left(\mathrm{E} \exp \left\{q_{1} \lambda \max _{v \leq u ; u, v \in V_{\varepsilon_{1}}}\left(X_{f}(u)-X_{f}(v)\right)\right\}\right)^{1 / q_{1}} \\
& \times \prod_{k=2}^{m}\left(\mathrm{E} \exp \left\{q_{k} \lambda \max _{v \leq u ; u, v \in V_{\varepsilon_{k}}}(K(u, v))\right\}\right)^{1 / q_{k}} \\
\leq & \left(\mathrm{E} \exp \left\{q_{1} \lambda \max _{v \leq u ; u, v \in V_{\varepsilon_{1}}}\left(X_{f}(u)-X_{f}(v)\right)\right\}\right)^{1 / q_{1}} \\
& \times \prod_{k=2}^{\infty}\left(\mathrm{E} \exp \left\{q_{k} \lambda \max _{v \leq u ; u, v \in V_{\varepsilon_{k}}}(K(u, v))\right\}\right)^{1 / q_{k}} \\
= & \left(J_{1}\right)^{1 / q_{1}} \cdot \prod_{k=2}^{\infty}\left(J_{k}\right)^{1 / q_{k}}
\end{aligned}
$$

for all $\lambda>0$, where

$$
K(u, v)=X_{f}(u)-X_{f}\left(\alpha_{k-1}(u)\right)-X_{f}(v)+X_{f}\left(\alpha_{k-1}(v)\right) .
$$

Each term on the right hand side of (14) is considered separately. Theorem 1.1 and assumption (6) imply

$$
\begin{aligned}
\mathrm{E} \exp \left\{q_{1} \lambda(X(u)-X(v))\right\} & \leq \exp \left\{\varphi\left(q_{1} \lambda \tau_{\varphi}(X(u)-X(v))\right)\right\} \\
& \leq \exp \left\{\varphi\left(q_{1} \lambda \sigma(\rho(u, v))\right)\right\}
\end{aligned}
$$


Then we use the condition $|f(u)-f(v)| \leq \delta(\rho(u, v))$ and obtain

$$
\begin{aligned}
J_{1} & \leq \sum_{v \leq u ; u, v \in V_{\varepsilon_{1}}} \operatorname{Exp}\left\{q_{1} \lambda(X(u)-X(v))\right\} \exp \left\{q_{1} \lambda(f(v)-f(u))\right\} \\
& \leq \sum_{v \leq u ; u, v \in V_{\varepsilon_{1}}} \exp \left\{\varphi\left(q_{1} \lambda \sigma(\rho(v, u))\right)+q_{1} \lambda \delta(\rho(v, u))\right\} \\
& =\sum_{i=1}^{N\left(\varepsilon_{1}\right)} \sum_{j=1}^{i} \exp \left\{\varphi\left(q_{1} \lambda \sigma\left(2 \varepsilon_{1}(i-j)\right)\right)+q_{1} \lambda \delta\left(2 \varepsilon_{1}(i-j)\right)\right\} \\
& =\sum_{l=0}^{N\left(\varepsilon_{1}\right)-1}\left(N\left(\varepsilon_{1}\right)-l\right) \exp \left\{\varphi\left(q_{1} \lambda \sigma\left(2 \varepsilon_{1} l\right)\right)+q_{1} \lambda \delta\left(2 \varepsilon_{1} l\right)\right\} .
\end{aligned}
$$

Further, Theorem 1.1 and the Cauchy-Bunyakowskiu inequality together with assumption (6) prove that

(16)

$$
\begin{aligned}
& \mathrm{E} \exp \left\{q_{k} \lambda\left(X(u)-X\left(\alpha_{k-1}(u)\right)-X(v)+X\left(\alpha_{k-1}(v)\right)\right)\right\} \\
& \quad \leq\left(\mathrm{E} \exp \left\{2 q_{k} \lambda\left(X(u)-X\left(\alpha_{k-1}(u)\right)\right)\right\} \mathrm{E} \exp \left\{2 q_{k} \lambda\left(X\left(\alpha_{k-1}(v)\right)-X(v)\right)\right\}\right)^{1 / 2} \\
& \quad \leq \exp \left\{\varphi\left(2 q_{k} \lambda \sigma\left(\varepsilon_{k-1}\right)\right)\right\} .
\end{aligned}
$$

Then

$$
\begin{aligned}
J_{k} \leq & \sum_{v \leq u ; u, v \in V_{\varepsilon_{k}}} \operatorname{Eexp}\left\{q_{k} \lambda\left(X(u)-X\left(\alpha_{k-1}(u)\right)-X(v)+X\left(\alpha_{k-1}(v)\right)\right)\right\} \\
& \times \exp \left\{q_{k} \lambda\left(f(u)-f\left(\alpha_{k-1}(u)\right)\right)\right\} \exp \left\{q_{k} \lambda\left(f\left(\alpha_{k-1}(v)\right)-f(v)\right)\right\} \\
& \leq \frac{N\left(\varepsilon_{k}\right)^{2}+N\left(\varepsilon_{k}\right)}{2} \exp \left\{\varphi\left(2 q_{k} \lambda \sigma\left(\varepsilon_{k-1}\right)\right)+2 q_{k} \lambda \max _{u \in V_{\varepsilon_{k}}} \delta\left(\rho\left(u, \alpha_{k-1}(u)\right)\right)\right\} \\
& \leq L\left(\varepsilon_{k}\right) \exp \left\{\varphi\left(2 q_{k} \lambda \sigma\left(\varepsilon_{k-1}\right)\right)+2 q_{k} \lambda \delta\left(\varepsilon_{k-1}\right)\right\} .
\end{aligned}
$$

Applying inequalities (14)-(17) we complete the proof of Lemma 2.1.

Remark 2.1. Inequality (8) is nontrivial only if the product on its right hand side is finite. There are simpler ways to estimate the term $J_{1}$ in inequality (15). However, the bounds for $W(\lambda)$ defined by (9) become less sharp in such cases. One of the possible ways here is to use the inequality

$$
W(\lambda) \leq\left(L\left(\varepsilon_{1}\right)\right)^{1 / q_{1}} \exp \left\{\frac{1}{q_{1}} \varphi\left(q_{1} \lambda \sigma\left(2 \varepsilon_{1}\left(N\left(\varepsilon_{1}\right)-1\right)\right)\right)+\lambda \delta\left(2 \varepsilon_{1}\left(N\left(\varepsilon_{1}\right)-1\right)\right)\right\} .
$$

Another possibility is based on the bound

$$
W(\lambda)^{q_{1}} \leq \int_{0}^{N\left(\varepsilon_{1}\right)}\left(N\left(\varepsilon_{1}\right)-x\right) \exp \left\{\varphi\left(q_{1} \lambda \sigma\left(2 \varepsilon_{1} x\right)\right)+q_{1} \lambda \delta\left(2 \varepsilon_{1} x\right)\right\} d x .
$$

Theorem 2.1. Assume that all the assumptions of Lemma 2.1 hold. Then

$$
\begin{aligned}
& \mathrm{P}\left\{\sup _{s \leq t ; s, t \in B}(X(t)-X(s)-(f(t)-f(s)))>x\right\} \leq Z(x), \\
& \mathrm{P}\left\{\inf _{s \leq t ; s, t \in B}(X(t)-X(s)-(f(t)-f(s)))<-x\right\} \leq Z(x), \\
& \mathrm{P}\left\{\sup _{s \leq t ; s, t \in B}|X(t)-X(s)-(f(t)-f(s))|>x\right\} \leq 2 Z(x)
\end{aligned}
$$


for all $x>0$, where

$$
\begin{aligned}
Z(x)=\prod_{k=2}^{\infty}( & \left.L\left(\varepsilon_{k}\right)\right)^{1 / q_{k}} \\
& \quad \times \inf _{\lambda>0} W(\lambda) \exp \left\{\sum_{k=2}^{\infty}\left(\frac{1}{q_{k}} \varphi\left(2 q_{k} \lambda \sigma\left(\varepsilon_{k-1}\right)\right)+2 \lambda \delta\left(\varepsilon_{k-1}\right)\right)-\lambda x\right\}
\end{aligned}
$$

and where $W(\lambda)$ is defined by (9).

Proof. The proof of Theorem 2.1 follows directly from the preceding Lemma 2.1 and the Chebyshev inequality.

Let $p \in(0,1)$ and $q_{1}=v$, where $v$ is a number such that $v \geq(1-p)^{-1}$, whence $q_{1}>1$. Put

$$
\begin{gathered}
q_{k}=\frac{1}{2 \lambda \beta p^{k-1}} \varphi^{(-1)}\left(\varphi\left(\frac{2 \lambda \beta}{1-p}\right)+\ln \left(\frac{N\left(\varepsilon_{k}\right)^{2}+N\left(\varepsilon_{k}\right)}{2}\right)\right), \quad k=2,3, \ldots, \\
\varepsilon_{k}=\sigma^{(-1)}\left(\beta p^{k}\right), \quad p \in(0,1), \quad k=0,1,2, \ldots
\end{gathered}
$$

Then

$$
q_{k} \geq \frac{1}{p^{k-1}(1-p)}>1
$$

and

$$
\frac{1}{q_{k}} \leq \frac{2 \lambda \beta p^{k-1}}{\varphi^{(-1)}\left(\varphi\left(\frac{2 \lambda \beta}{1-p}\right)\right)}=p^{k-1}(1-p), \quad k=2,3, \ldots
$$

that is

$$
\sum_{k=1}^{\infty} \frac{1}{q_{k}} \leq \sum_{k=1}^{\infty} p^{k-1}(1-p)=1
$$

This means that the sequence $\left\{q_{k}\right\}$ defined above satisfies assumptions of Lemma 2.1, Applying inequality (8) with this sequence $\left\{q_{k}\right\}$, we prove the following result.

Lemma 2.2. Assume that condition (6) holds for a stochastic process

$$
X(t)=\{X(t), t \in B\}
$$

belonging to the class $V(\varphi, \psi)$. Let $f=\{f(t), t \in B\}$ be a continuous function such that

$$
|f(u)-f(w)| \leq \delta(\rho(u, w)),
$$

where $\delta=\{\delta(s), s>0\}$ is a nonnegative increasing function, and let

$$
\int_{0}^{\beta} \zeta_{\varphi}\left(\ln \left(L\left(\sigma^{(-1)}(u)\right)\right)\right) d u<\infty .
$$

Then

$$
\begin{aligned}
E \exp & \left\{\lambda \sup _{s \leq t ; s, t \in B}(X(t)-X(s)-(f(t)-f(s)))\right\} \\
\leq & W_{1}(\lambda, p) \exp \left\{\varphi\left(\frac{2 \lambda \beta}{1-p}\right) p+\frac{4 \lambda}{p(1-p)} \int_{0}^{\beta p^{2}} \zeta_{\varphi}\left(\ln \left(L\left(\sigma^{(-1)}(u)\right)\right)\right) d u\right\} \\
& \times \exp \left\{2 \lambda \sum_{k=1}^{\infty} \delta\left(\sigma^{(-1)}\left(\beta p^{k}\right)\right)\right\}
\end{aligned}
$$


for all $\lambda>0$ and $p \in(0,1)$, where

$$
\begin{aligned}
W_{1}(\lambda, p)=\inf _{v \geq \frac{1}{1-p}}( & \sum_{l=0}^{N\left(\sigma^{(-1)}(\beta p)\right)-1}\left(N\left(\sigma^{(-1)}(\beta p)\right)-l\right) \\
& \left.\times \exp \left\{\varphi\left(\lambda v \sigma\left(2 l \sigma^{(-1)}(\beta p)\right)\right)+\lambda v \delta\left(2 l \sigma^{(-1)}(\beta p)\right)\right\}\right)^{1 / v} .
\end{aligned}
$$

Proof. We use Lemma 2.1] with the sequences $\left\{q_{k}\right\}$ and $\left\{\varepsilon_{k}\right\}$ defined by (24) and (25), respectively. Consider the sum

$$
\tilde{Z}=\sum_{k=2}^{\infty} \frac{1}{q_{k}}\left(\ln \left(L\left(\varepsilon_{k}\right)\right)+\varphi\left(2 \lambda \beta p^{k-1} q_{k}\right)\right) .
$$

Then

$$
\begin{aligned}
\tilde{Z} & =\sum_{k=2}^{\infty} \frac{1}{q_{k}} \ln \left(L\left(\varepsilon_{k}\right)\right)+\sum_{k=2}^{\infty} \frac{1}{q_{k}} \varphi\left(2 \lambda \beta p^{k-1} \frac{\varphi^{(-1)}\left(\varphi\left(\frac{2 \lambda \beta}{1-p}\right)+\ln \left(L\left(\varepsilon_{k}\right)\right)\right)}{2 \lambda \beta p^{k-1}}\right) \\
& =2 \sum_{k=2}^{\infty} \frac{1}{q_{k}} \ln \left(L\left(\varepsilon_{k}\right)\right)+\varphi\left(\frac{2 \lambda \beta}{1-p}\right) \sum_{k=2}^{\infty} \frac{1}{q_{k}} \\
& \leq 2 \sum_{k=2}^{\infty} \ln \left(L\left(\varepsilon_{k}\right)\right) \frac{2 \lambda \beta p^{k-1}}{\varphi^{(-1)}\left(\ln \left(L\left(\varepsilon_{k}\right)\right)\right)}+\varphi\left(\frac{2 \lambda \beta}{1-p}\right) \sum_{k=2}^{\infty} p^{k-1}(1-p) \\
& =\varphi\left(\frac{2 \lambda \beta}{1-p}\right) p+4 \lambda \sum_{k=2}^{\infty} \zeta_{\varphi}\left(\ln \left(L\left(\sigma^{(-1)}\left(\beta p^{k}\right)\right)\right)\right) \beta p^{k-1} .
\end{aligned}
$$

The function $\varphi(x) / x$ increases for $x>0$, thus the function $\zeta_{\varphi}(x)=x / \varphi^{(-1)}(x)$ increases for $x>0$, too. Hence

$$
\int_{\beta p^{k+1}}^{\beta p^{k}} \zeta_{\varphi}\left(\ln \left(L\left(\sigma^{(-1)}(u)\right)\right)\right) d u \geq \zeta_{\varphi}\left(\ln \left(L\left(\sigma^{(-1)}\left(\beta p^{k}\right)\right)\right)\right) \beta p^{k}(1-p) .
$$

It follows from (29) and (30) that

$$
\tilde{Z} \leq \varphi\left(\frac{2 \lambda \beta}{1-p}\right) p+\frac{4 \lambda}{p(1-p)} \int_{0}^{\beta p^{2}} \zeta_{\varphi}\left(\ln \left(L\left(\sigma^{(-1)}(u)\right)\right)\right) d u .
$$

Therefore inequality (27) follows from (8) and (31).

Remark 2.2. Similar to Remark 2.1, the corresponding term in (28) can be estimated in at least two other ways. Namely, either

$$
\begin{aligned}
& W_{1}(\lambda, p) \leq \inf _{v \geq \frac{1}{1-p}}(L\left(\sigma^{(-1)}(\beta p)\right) \\
& \times \exp \left\{\varphi\left(\lambda v \sigma\left(2 \sigma^{(-1)}(\beta p)\left(N\left(\sigma^{(-1)}(\beta p)\right)-1\right)\right)\right)\right. \\
&\left.\left.\quad+\lambda v \delta\left(2 \sigma^{(-1)}(\beta p)\left(N\left(\sigma^{(-1)}(\beta p)\right)-1\right)\right)\right\}\right)^{1 / v}
\end{aligned}
$$


or

$$
\begin{aligned}
W_{1}(\lambda, p) \leq \inf _{v \geq \frac{1}{1-p}}\left(\int_{0}^{N\left(\sigma^{(-1)}(\beta p)\right)}(\right. & \left.N\left(\sigma^{(-1)}(\beta p)\right)-x\right) \\
\times \exp \left\{\varphi\left(\lambda v \sigma\left(2 \sigma^{(-1)}(\beta p) x\right)\right)\right. & \left.\left.+\lambda v \delta\left(2 \sigma^{(-1)}(\beta p) x\right)\right\} d x\right)^{1 / v} .
\end{aligned}
$$

Using the same sequences of real numbers $\left\{q_{k}\right\}$ and $\left\{\varepsilon_{k}\right\}$ as in Lemma 2.2, we obtain the following result.

Theorem 2.2. Assume that condition (6) holds for a stochastic process

$$
X(t)=\{X(t), t \in B\}
$$

belonging to the class $V(\varphi, \psi)$. Let $f=\{f(t), t \in B\}$ be a continuous function such that

$$
|f(u)-f(w)| \leq \delta(\rho(u, w))
$$

where $\delta=\{\delta(s), s>0\}$ is a nonnegative increasing function. Let $r=\{r(u): u \geq 1\}$ be a continuous function such that $r(1)=0, r(u)>0$ for $u>1$, and that the function $s(t)=r(\exp \{t\}), t \geq 0$, be convex. If

$$
\int_{0}^{\beta} \frac{r\left(L\left(\sigma^{(-1)}(u)\right)\right)}{\varphi^{(-1)}\left(\ln \left(L\left(\sigma^{(-1)}(u)\right)\right)\right)} d u<\infty
$$

for all $p \in(0 ; 1)$ and $x>0$, then

where

$$
\begin{aligned}
& \mathrm{P}\left\{\sup _{s \leq t ; s, t \in B}(X(t)-X(s)-(f(t)-f(s)))>x\right\} \leq Z_{r}(p, x), \\
& \mathrm{P}\left\{\inf _{s \leq t ; s, t \in B}(X(t)-X(s)-(f(t)-f(s)))<-x\right\} \leq Z_{r}(p, x), \\
& \mathrm{P}\left\{\sup _{s \leq t ; s, t \in B}|X(t)-X(s)-(f(t)-f(s))|>x\right\} \leq 2 Z_{r}(p, x),
\end{aligned}
$$

$$
\begin{aligned}
Z_{r}(p, x)=\inf _{\lambda>0} & W_{1}(\lambda, p) \exp \left\{p \varphi\left(\frac{2 \lambda \beta}{1-p}\right)+\lambda\left(2 \sum_{k=1}^{\infty} \delta\left(\sigma^{(-1)}\left(\beta p^{k}\right)\right)-x\right)\right\} \\
& \times\left(r^{(-1)}\left(\frac{\lambda}{p(1-p)} \int_{0}^{\beta p^{2}} \frac{r\left(L\left(\sigma^{(-1)}(u)\right)\right)}{\varphi^{(-1)}\left(\ln \left(L\left(\sigma^{(-1)}(u)\right)\right)\right.} d u\right)\right)
\end{aligned}
$$

and where $W_{1}(\lambda, p)$ is defined by equality (28).

Proof. Theorem 2.2 follows from Lemmas 2.1 and 2.2. The proof is similar to that of Theorem 3.2 in [7] or to that of Theorem 3.4 in [4].

Using the sequence $q_{k}=(1-p)^{-1} p^{1-k}, k=1,2, \ldots$, in inequality (8) of Lemma 2.1 we obtain the following result whose assumptions are easier to check than those of Theorem 2.2 .

Theorem 2.3. Assume that assumption (66) holds for a stochastic process

$$
X(t)=\{X(t), t \in B\}
$$

belonging to the class $V(\varphi, \psi)$. Let $f=\{f(t), t \in B\}$ be a continuous function such that

$$
|f(u)-f(w)| \leq \delta(\rho(u, w))
$$


where $\delta=\{\delta(s), s>0\}$ is a nonnegative increasing function. Let $r_{1}=\left\{r_{1}(u), u \geq 1\right\}$ be a continuous function such that $r(u)>0$ for $u>1$ and that $s(t)=r(\exp \{t\}), t \geq 0$, be a convex function. If

$$
\int_{0}^{\beta} r\left(L\left(\sigma^{(-1)}(u)\right)\right) d u<\infty
$$

then

$$
\begin{aligned}
& \mathrm{P}\left\{\sup _{s \leq t ; s, t \in B}(X(t)-X(s)-(f(t)-f(s)))>x\right\} \leq Z_{r_{1}}(p, x), \\
& \mathrm{P}\left\{\inf _{s \leq t ; s, t \in B}(X(t)-X(s)-(f(t)-f(s)))<-x\right\} \leq Z_{r_{1}}(p, x), \\
& \mathrm{P}\left\{\sup _{s \leq t ; s, t \in B}|X(t)-X(s)-(f(t)-f(s))|>x\right\} \leq 2 Z_{r_{1}}(p, x)
\end{aligned}
$$

for all $p \in(0 ; 1)$ and $x>0$, where

$$
\begin{aligned}
Z_{r_{1}}(p, t, x)= & r^{(-1)}\left(\frac{1}{\beta p} \int_{0}^{\beta p^{2}} r\left(L\left(\sigma^{(-1)}(u)\right)\right) d u\right) \\
& \times \inf _{\lambda>0} W_{2}(\lambda, p) \exp \left\{p \varphi\left(\frac{2 \lambda \beta}{1-p}\right)+\lambda\left(2 \sum_{k=1}^{\infty} \delta\left(\sigma^{(-1)}\left(\beta p^{k}\right)\right)-x\right)\right\}, \\
W_{2}(\lambda, p)=\left(\sum_{l=0}^{N\left(\sigma^{(-1)}(\beta p)\right)-1}\left(N\left(\sigma^{(-1)}(\beta p)\right)-l\right)\right. & \left.\times \exp \left\{\varphi\left(\frac{\lambda \sigma\left(2 l \sigma^{(-1)}(\beta p)\right)}{1-p}\right)+\frac{\lambda \delta\left(2 l \sigma^{(-1)}(\beta p)\right)}{1-p}\right\}\right)^{1-p} .
\end{aligned}
$$

Proof. Theorem 2.3 follows from Lemma 2.1. The proof is similar to that of Theorem 3.1 in [7] or to that of Theorem 3.5 in [4].

Now we use the sequence

$$
q_{k}=e^{p} \frac{(k-1) !}{p^{k-1}}, \quad p \in(0,1), k=1,2, \ldots,
$$

in inequality (8) of Lemma 2.1.

Then $q_{k}>1$ and

$$
\sum_{k=1}^{\infty} \frac{1}{q_{k}}=e^{-p} \sum_{k=1}^{\infty} \frac{p^{k-1}}{(k-1) !}=1 .
$$

Thus this sequence $\left\{q_{k}\right\}$ satisfies the assumption of Lemma 2.1, Using inequality (8) of Lemma 2.1 with the same sequence $\left\{q_{k}\right\}$, we obtain the following result.

Lemma 2.3. Assume that condition (6) holds for a stochastic process

$$
X(t)=\{X(t), t \in B\}
$$

belonging to the class $V(\varphi, \psi)$. Let $f=\{f(t), t \in B\}$ be a continuous function such that

$$
|f(u)-f(w)| \leq \delta(\rho(u, w)),
$$

where $\delta=\{\delta(s), s>0\}$ is a nonnegative increasing function, and let

$$
\int_{0}^{\beta} \ln \left(L\left(\sigma^{(-1)}(u)\right)\right) d u<\infty .
$$


Then

$$
\begin{aligned}
\mathrm{E} & \exp \left\{\lambda \sup _{s \leq t ; s, t \in B}(X(t)-X(s)-(f(t)-f(s)))\right\} \\
\leq & W_{2}(\lambda, p) \exp \left\{\left(1-e^{-p}\right) \varphi\left(2 \lambda \beta e^{p}\right)+\frac{1}{\beta e^{p}\left(1-\frac{p}{2}\right)} \int_{0}^{\beta p} \ln \left(L\left(\sigma^{(-1)}(u)\right)\right) d u\right\} \\
& \times \exp \left\{2 \lambda \sum_{k=1}^{\infty} \delta\left(\sigma^{(-1)}\left(\frac{\beta p^{k}}{k !}\right)\right)\right\}
\end{aligned}
$$

for all $\lambda>0$ and $p \in(0,1)$, where $W_{2}(\lambda, p)$ is defined by (36).

Proof. We use the sequence of real numbers $\left\{q_{k}\right\}$ defined by (37) in Lemma 2.1. Consider the sum

$$
\tilde{Z}=\sum_{k=2}^{\infty} \frac{1}{q_{k}}\left(\ln \left(L\left(\varepsilon_{k}\right)\right)+\varphi\left(2 \lambda q_{k} \sigma\left(\varepsilon_{k-1}\right)\right)\right)
$$

Putting

$$
\varepsilon_{k}=\sigma^{(-1)}\left(\frac{\beta p^{k}}{k !}\right)
$$

we get

$$
\begin{aligned}
\tilde{Z} & =\sum_{k=2}^{\infty} \frac{1}{q_{k}} \ln \left(L\left(\varepsilon_{k}\right)\right)+\sum_{k=2}^{\infty} \frac{1}{q_{k}} \varphi\left(2 \lambda e^{p} \frac{(k-1) !}{p^{k-1}} \frac{\beta p^{k-1}}{(k-1) !}\right) \\
& =\sum_{k=2}^{\infty} \frac{\ln \left(L\left(\sigma^{(-1)}\left(\frac{\beta p^{k}}{k !}\right)\right)\right) p^{k-1}}{e^{p}(k-1) !}+\varphi\left(2 \lambda \beta e^{p}\right) e^{-p} \sum_{k=2}^{\infty} \frac{p^{k-1}}{(k-1) !} \\
& =\left(1-e^{-p}\right) \varphi\left(2 \lambda \beta e^{p}\right)+e^{-p} \sum_{k=2}^{\infty} \frac{\ln \left(L\left(\sigma^{(-1)}\left(\frac{\beta p^{k}}{k !}\right)\right)\right) p^{k-1}}{(k-1) !} .
\end{aligned}
$$

Since $\ln \left(L\left(\sigma^{(-1)}(u)\right)\right)$ is a decreasing function for $u>0$,

$$
\begin{aligned}
& \int_{\frac{\beta p^{k}}{k !}}^{\frac{\beta p^{k-1}}{(k-1) !}} \ln \left(L\left(\sigma^{(-1)}(u)\right)\right) d u \\
& \quad \geq \ln \left(L\left(\sigma^{(-1)}\left(\frac{\beta p^{k}}{k !}\right)\right)\right)\left(\frac{\beta p^{k-1}}{(k-1) !}-\frac{\beta p^{k}}{k !}\right) \\
& \quad=\ln \left(L\left(\sigma^{(-1)}\left(\frac{\beta p^{k}}{k !}\right)\right)\right) \frac{\beta p^{k-1}}{(k-1) !}\left(1-\frac{p}{2}\right), \quad k \geq 2 .
\end{aligned}
$$

Now we get from (41) and (42) that

$$
\tilde{Z} \leq\left(1-e^{-p}\right) \varphi\left(2 \lambda \beta e^{p}\right)+\frac{1}{\beta e^{p}\left(1-\frac{p}{2}\right)} \int_{0}^{\beta p} \ln \left(L\left(\sigma^{(-1)}(u)\right)\right) d u .
$$

Therefore inequality (39) follows from (8) and (43).

Using the sequences $\left\{q_{k}\right\}$ and $\left\{\varepsilon_{k}\right\}$ defined by (37) and (40), respectively, Lemma 2.3 implies the following estimate for the distribution of the supremum of increments of the process $X_{f}(t)=X(t)-f(t)$. 
Theorem 2.4. Assume that condition (6) holds for a stochastic process

$$
X(t)=\{X(t), t \in B\}
$$

belonging to the class $V(\varphi, \psi)$. Let $f=\{f(t), t \in B\}$ be a continuous function such that

$$
|f(u)-f(w)| \leq \delta(\rho(u, w))
$$

where $\delta=\{\delta(s), s>0\}$ is a nonnegative increasing function. Let

$$
r=\{r(u), u \geq 1\}
$$

be a continuous function such that $r(u)>0$ for $u>1$ and let $s(t)=r(\exp \{t\}), t \geq 0$, be a convex function. If

$$
\int_{0}^{\beta} r\left(L\left(\sigma^{(-1)}(u)\right)\right) d u<\infty
$$

then

$$
\begin{aligned}
& \mathrm{P}\left\{\sup _{s \leq t ; s, t \in B}(X(t)-X(s)-(f(t)-f(s)))>x\right\} \leq Z_{r_{2}}(p, x), \\
& \mathrm{P}\left\{\inf _{s \leq t ; s, t \in B}(X(t)-X(s)-(f(t)-f(s)))<-x\right\} \leq Z_{r_{2}}(p, x), \\
& \mathrm{P}\left\{\sup _{s \leq t ; s, t \in B}|X(t)-X(s)-(f(t)-f(s))|>x\right\} \leq 2 Z_{r_{2}}(p, x)
\end{aligned}
$$

for all $p \in(0 ; 1)$ and $x>0$, where

$$
\begin{aligned}
Z_{r_{2}}(p, x)= & r^{(-1)}\left(\frac{1}{\beta e^{p}\left(1-\frac{p}{2}\right)} \int_{0}^{\beta p} r\left(L\left(\sigma^{(-1)}(u)\right)\right) d u\right) \\
& \times \inf _{\lambda>0} W_{2}(\lambda, p) \exp \left\{p \varphi\left(\frac{2 \lambda \beta}{1-p}\right)+\lambda\left(2 \sum_{k=1}^{\infty} \delta\left(\sigma^{(-1)}\left(\beta p^{k}\right)\right)-x\right)\right\}
\end{aligned}
$$

and where $W_{2}(\lambda, p)$ is defined in equality (36) .

Proof. We follow the lines of the proof of Lemma 2.3. Since $s(t)=r(\exp \{t\})$ is a convex function, we have

$$
s\left(\sum_{i=1}^{\infty} \Delta_{i} x_{i}\right) \leq \sum_{i=1}^{\infty} \Delta_{i} s\left(x_{i}\right)
$$

for all $x_{i} \geq 0$ and all $\Delta_{i}>0, i=1, \ldots, \infty$, such that

$$
\sum_{i=1}^{\infty} \Delta_{i}=1
$$


Consider

$$
\begin{aligned}
& \exp \left\{\sum_{k=2}^{\infty} \frac{\ln \left(L\left(\sigma^{(-1)}\left(\frac{\beta p^{k}}{k !}\right)\right)\right) p^{k-1}}{e^{p}(k-1) !}\right\} \\
& \quad=r^{(-1)}\left(s\left(\sum_{k=2}^{\infty} \frac{\left.\left.\ln \left(L\left(\sigma^{(-1)}\left(\frac{\beta p^{k}}{k !}\right)\right)\right) p^{k-1}\right)\right)}{e^{p}(k-1) !}\right)\right. \\
& \leq r^{(-1)}\left(\sum_{k=2}^{\infty} \frac{p^{k-1}}{e^{p}(k-1) !} s\left(\ln \left(L\left(\sigma^{(-1)}\left(\frac{\beta p^{k}}{k !}\right)\right)\right)\right)\right) \\
& \leq r^{(-1)}\left(\frac{1}{\beta e^{p}\left(1-\frac{p}{2}\right)} \int_{0}^{\beta p} r\left(L\left(\sigma^{(-1)}(u)\right)\right) d u\right) .
\end{aligned}
$$

Thus Theorem 2.4 follows from Lemmas 2.1 and 2.3 by applying estimates (45) and Chebyshev's inequality.

\section{An application to the Generalized fractional Brownian motion}

Assume that $X(t)=\{X(t), t \in[a, b]\}$ is a separable sub-Gaussian stochastic process defined in the interval $[a, b],-\infty<a<b<\infty$. Assume that, for some $H \in(0,1)$,

$$
\tau(X(t)-X(s)) \leq|t-s|^{H},
$$

that is, the process $X(t)$ belongs to the class $V(\varphi, \varphi)$ with $\varphi(x)=x^{2} / 2, x \geq 0$. Note that condition (46) holds, in particular, for the (Gaussian) fractional Brownian motion with the corresponding Hurst index $H$. More details on the generalized fractional Brownian motion belonging to the classes $V(\varphi, \psi)$ are given in the papers [3, 6, 8].

Assume that $f(t)$ is a continuous function defined in the interval $[a, b]$ and such that

$$
|f(t)-f(s)| \leq c|t-s|^{n},
$$

where $c>0$ and $n>0$ are some constants. The bounds collected in the result below follow from Theorem 2.3 .

Theorem 3.1. Assume that conditions (46) and (47) hold for a sub-Gaussian stochastic process $X(t)=\{X(t), t \in[a, b]\}$ and a function $f=\{f(t), t \in[a, b]\}$. Then

$$
\begin{aligned}
& \mathrm{P}\left\{\sup _{s \leq t ; s, t \in B}(X(t)-X(s)-(f(t)-f(s)))>x\right\} \leq Z(p, x), \\
& \mathrm{P}\left\{\inf _{s \leq t ; s, t \in B}(X(t)-X(s)-(f(t)-f(s)))<-x\right\} \leq Z(p, x), \\
& \mathrm{P}\left\{\sup _{s \leq t ; s, t \in B}|X(t)-X(s)-(f(t)-f(s))|>x\right\} \leq 2 Z(p, x)
\end{aligned}
$$

for all

$$
p \in\left(0 ;\left(\frac{2}{3}\right)^{H}\right]
$$

and

$$
x>c(b-a)^{n}+\frac{2 c\left(\beta p^{2}\right)^{n / H}}{1-p^{n / H}},
$$


where

$$
\begin{aligned}
Z(p, x)= & \frac{(b-a)^{2}}{2}(\beta p e)^{2 / H} \\
& \times\left(\sum_{l=0}^{N\left((\beta p)^{1 / H}\right)-1}\left(\frac{b-a}{2(\beta p)^{1 / H}}+1-l\right)\right. \\
& \left.\times \exp \left\{-\frac{\left(x-c(2 l)^{n}(\beta p)^{n / H}-\frac{2 c\left(\beta p^{2}\right)^{n / H}}{1-p^{n / H}}\right)^{2}}{2(2 l)^{2 H}(\beta p)^{2}+\frac{8 p \beta^{2}}{1-p}}\right\}\right)^{1-p} .
\end{aligned}
$$

Proof. Consider the function

$$
r(u)=u^{\alpha}, \quad 0<\alpha<\frac{H}{2} .
$$

If $p$ does not exceed $\left(\frac{2}{3}\right)^{H}$, then

$$
\frac{b-a}{2 u^{1 / H}}>\frac{3}{2}
$$

since

$$
u \leq\left(\frac{2}{3}\right)^{H}\left(\frac{b-a}{2}\right)^{H} \leq p \beta .
$$

It is clear that

$$
\begin{aligned}
r^{(-1)} & \left(\frac{1}{\beta p} \int_{0}^{\beta p^{2}} r\left(L\left(\sigma^{(-1)}(u)\right)\right) d u\right) \\
& \leq\left(\frac{1}{\beta p} \int_{0}^{\beta p^{2}}\left(\left(\frac{b-a}{2 u^{1 / H}}+1\right)^{2}+\frac{b-a}{2 u^{1 / H}}+1\right)^{\alpha} / 2^{\alpha} d u\right)^{1 / \alpha} \\
& \leq \frac{1}{2}\left(\frac{1}{\beta p} \int_{0}^{\beta p^{2}}\left(\frac{b-a}{2 u^{1 / H}}+\frac{3}{2}\right)^{2 \alpha} d u\right)^{1 / \alpha} \\
& <\frac{1}{2}\left(\frac{1}{\beta p} \int_{0}^{\beta p}\left(\frac{b-a}{u^{1 / H}}\right)^{2 \alpha} d u\right)^{1 / \alpha} \\
& =\frac{(b-a)^{2}}{2}(\beta p)^{2 / H}\left(1-\frac{2 \alpha}{H}\right)^{-1 / \alpha} \\
& \rightarrow \frac{(b-a)^{2}}{2}(\beta p e)^{2 / H}, \quad \alpha \rightarrow 0,
\end{aligned}
$$

and

$$
\sum_{k=1}^{\infty} \delta\left(\sigma^{(-1)}\left(\beta p^{k}\right)\right)=\sum_{k=1}^{\infty} c\left(\beta p^{k}\right)^{n / H}=\frac{c \beta^{n / H} p^{n / H}}{1-p^{n / H}}
$$


Thus

$$
\begin{aligned}
& \inf _{\lambda>0} \exp \left\{p \varphi\left(\frac{2 \lambda \beta}{1-p}\right)+\lambda\left(2 \sum_{k=1}^{\infty} \delta\left(\sigma^{(-1)}\left(\beta p^{k}\right)\right)-x\right)\right\} W_{2}(\lambda, p) \\
& =\inf _{\lambda>0} \exp \left\{\frac{2 p \lambda^{2} \beta^{2}}{(1-p)^{2}}+\lambda\left(\frac{2 c \beta^{n / H} p^{n / H}}{1-p^{n / H}}-x\right)\right\} \\
& N\left((\beta p)^{1 / H}\right)-1 \\
& \times\left(\sum_{l=0}^{N((\beta p)}\left(N\left((\beta p)^{1 / H}\right)-l\right)\right. \\
& \left.\times \exp \left\{\frac{\lambda^{2}(2 l)^{2 H}(\beta p)^{2}}{2(1-p)^{2}}+\frac{\lambda(2 l)^{n} c(\beta p)^{n / H}}{1-p}\right\}\right)^{1-p} \\
& \leq\left(\sum_{l=0}^{N\left((\beta p)^{1 / H}\right)-1}\left(\frac{b-a}{2(\beta p)^{1 / H}}+1-l\right)\right. \\
& \times \inf _{\lambda>0} \exp \left\{\lambda^{2}\left(\frac{2 p \beta^{2}}{(1-p)^{3}}+\frac{(2 l)^{2 H}(\beta p)^{2}}{2(1-p)^{2}}\right)\right. \\
& \left.\left.-\lambda\left(\frac{x-\frac{2 c \beta^{n / H} p^{n / H}}{1-p^{n / H}}}{1-p}-\frac{(2 l)^{n} c(\beta p)^{n / H}}{1-p}\right)\right\}\right)^{1-p} \\
& \leq\left(\sum_{l=0}^{N\left((\beta p)^{1 / H}\right)-1}\left(\frac{b-a}{2(\beta p)^{1 / H}}+1-l\right)\right. \\
& \left.\times \exp \left\{-\frac{\left(x-c(2 l)^{n}(\beta p)^{n / H}-\frac{2 c\left(\beta p^{2}\right)^{n / H}}{1-p^{n / H}}\right)^{2}}{2(2 l)^{2 H}(\beta p)^{2}+\frac{8 p \beta^{2}}{1-p}}\right\}\right)^{1-p} .
\end{aligned}
$$

Applying (49) and Theorem 2.3, we complete the proof of Theorem 3.1]

\section{CONCLUding REMARKS}

Properties of stochastic processes with $\varphi$-sub-Gaussian increments were studied in the paper. In particular, we studied the processes belonging to the class $V(\varphi, \psi), \varphi \prec \psi$. We obtained some bounds for the distribution of some extremal functionals of the increments of such processes. Among those functionals are, for example,

$$
\begin{aligned}
& \sup _{s \leq t ; s, t \in B}(X(t)-X(s)-(f(t)-f(s))), \\
& \inf _{s \leq t ; s, t \in B}(X(t)-X(s)-(f(t)-f(s))),
\end{aligned}
$$

where $f(t)$ is a continuous increasing function.

The general results obtained in the paper can be applied to a wide class of stochastic processes, to Gaussian processes, for example. A particular case of a generalized fractional Brownian motion is considered in the paper.

\section{BIBLIOGRAPHY}

1. R. Addie, P. Mannersalo, and I. Norros, Most probable paths and performance formulae for buffers with Gaussian input traffic, Eur. Trans. Telecommun. 13(3) (2002), 183-196. 
2. V. V. Buldygin and Yu. V. Kozachenko, Metric Characterization of Random Variables and Random Processes, "TBiMC", Kiev, 1998; English transl., American Mathematical Society, Providence, RI, 2000. MR.1743716 (2001g:60089)

3. Yu. V. Kozachenko, T. Sottinen, and O. I. Vasylyk, Self-similar processes with stationary increments in the spaces $\operatorname{SSub}_{\varphi}(\Omega)$, Teor. Imovir. Mat. Stat. 65 (2001), 67-78; English transl. in Theory Probab. Math. Statist. 65 (2002), 77-88. MR.1936131 (2004a:60078)

4. Yu. Kozachenko, O. Vasylyk, and R. Yamnenko, Upper estimate of overrunning by $\operatorname{Sub}_{\varphi}(\Omega)$ random process the level specified by continuous function, Random Oper. Stoch. Equ. 13 (2005), no. 2, 111-128. MR2152102(2006b:60207)

5. I. Norros, On the use of fractional Brownian motions in the theory of connectionless networks, IEEE Journal on Selected Areas in Communications 13 (1995), no. 6, 953-962.

6. R. Yamnenko, Ruin probability for generalized $\varphi$-sub-Gaussian fractional Brownian motion, Theory Stoch. Process. 12(28) (2006), no. 1-2, 261-275. MR2316577 (2008g:60109)

7. R. Yamnenko and O. Vasylyk, Random process from the class $V(\varphi, \psi)$ : exceeding a curve, Theory Stoch. Process. 13(29) (2007), no. 4, 219-232. MR2482262 (2010a:60125)

8. Yu. Kozachenko, O. I. Vasylyk, and R. E. Yamnenko, $\varphi$-sub-Gaussian Random Processes, "Kyiv University", Kyiv, 2008. (Ukrainian)

9. R. E. Yamnenko and O. S. Shramko, On the distribution of storage processes from the class $V(\varphi, \psi)$, Teor. Imovir. Mat. Stat. 83 (2010), 163-176; English transl. in Theory Probab. Math. Statist. 83 (2011), 191-206. MR2768858(2011j:60268)

Department of Probability Theory, Statistics, and Actuarial Mathematics, Faculty for Mechanics and Mathematics, National Taras Shevchenko University, Academician Glushkov Avenue, 2, Kiev 03127, Ukraine

E-mail address: yamnenko@univ.kiev.ua

Received 11/JUNE/2011

Translated by S. KVASKO 